\title{
Interferometric Signatures of Electron Transfer through Majorana Bound States
}

\author{
J. BARAŃSKI* AND T. ZIENKIEWICZ \\ Polish Air Force University, Dywizjonu 303, PL-08521 Dęblin, Poland
}

\begin{abstract}
In semiconducting nanowires deposited on top of $s$-wave superconductors, the combined effect of induced superconducting order, strong spin-orbit coupling, and magnetic field can lead to the realization of topologically non-trivial edge states. According to Kitayev's model, such states fulfill the axioms of Majorana quasiparticles. Recent development of quantum dot-nanowire hybrids provides useful tools to perform analysis which goes beyond mere observation of such states. One of the most challenging tasks is to demonstrate the nonlocal character of these states. Considering two quantum dots coupled to opposite edges of a chain hosting Majorana quasi-particles, we show that long range electron transfer through the Majorana states can be demonstrated by measurement of local currents. We show that the scattering of electron on one dot gives rise to interferometric signatures on the quantum dot connected to the opposite side of the nanowire.
\end{abstract}

DOI: 10.12693/APhysPolA.136.662

PACS/topics: 73.63.Kv, 73.23.Hk, 74.45.+c, 74.50.+r

\section{Introduction}

In famous "toy" model introduced by Alexei Kitaev [1], it was shown that one dimensional spinless $p$-wave superconducting chain can host two unpaired edge states which obey the axioms of Majorana quasiparticles. Predicted states are immune to local disturbances. High resistance to decoherences is provided by their nonlocal nature. Two edge states $\eta_{1}, \eta_{2}$ form one ordinary but highly nonlocal fermion $f=\frac{1}{\sqrt{2}}\left(\eta_{1}+\mathrm{i} \eta_{2}\right)$, and as a consequence local disturbances have no impact on Majorana zero modes [2]. This property makes Majorana quasiparticles good candidates for being a quantum information storage medium.

In recent years number of works have been addressed to realize the systems in which the fingerprints of such states can be detected [3-9]. In most widely explored realizations, semiconducting nanowire (e.g. InAs) with strong spin-orbit interaction is covered by a layer of $s$-wave superconductor (e.g. Al). In presence of magnetic field, for large enough Zeeman splitting, localized Majorana bound states (MBS) appear at the ends of the nanochain. In other realizations monoatomic chains of magnetic atoms are placed directly on top of polished superconductor with strong spin-orbit interaction $[6,7,9]$. Although experiments confirm existence of zero modes at the ends of such nanowires, the nonlocal nature of measured states is more elusive and signatures confirming this property have not been found yet. Some theoretical works have proposed the schemes to test nonlocality via crossed Andreev reflections [10-12]. Others, however, suggested that for vanishing overlap between edge

*corresponding author; e-mail: j.baranski@law.mil.pl states cross-correlations can not develop and the process is suppressed $[13,14]$. New proposals for testing of MBS nonlocality are highly requested.

Our idea is based on the measurement of features originating from the long-range electron scattering between two quantum dots (QDs) coupled to opposite edges of a topological chain. Scattering between two distant dots is possible due to the inherent nonlocal property of topological chain edge states. We predict that such scattering leads to the appearance of characteristic Fano features in QD's spectral function. We show that the manipulation of a "local" model parameters associated only to one of the dots, can cause a shift of interferometric signatures appearing in the second dot. We inspect the conditions at which features of such long-range scattering emerge in the spectral function of quantum dots.

\section{Formulation of the problem}

We analyze the structure schematically depicted in Fig. 1, where monoatomic chain of magnetic atoms (e.g. Fe atoms) is placed on top of $s$-wave superconductor (SC) with strong spin-orbit interaction (e.g. Pb surface). We assume that this nanowire is driven into a superconducting topological phase. In such conditions edge states of the nanowire can be represented by two topologically nontrivial zero-energy states $\eta_{1}$ and $\eta_{2}$ called Majorana modes. Due to the finite length of the nanowire, wavefunctions of these two states overlap on each other. The factor $\epsilon_{m}$ stands for tunneling rate between two edge states and it is proportional to $\mathrm{e}^{-\xi / L}$, where $\xi$ stands for superconductor's coherence length and $L$ is the length of the nanowire. In this work we use the term "topological chain" to describe nanowire with two Majorana modes induced on its opposite edges. In proposed architecture two quantum dots $\left(Q D_{1}, Q D_{2}\right)$ placed on top of SC substrate are tunnel-coupled to opposite edges of such topological chain. Experimentally such quantum dot-nanowire 


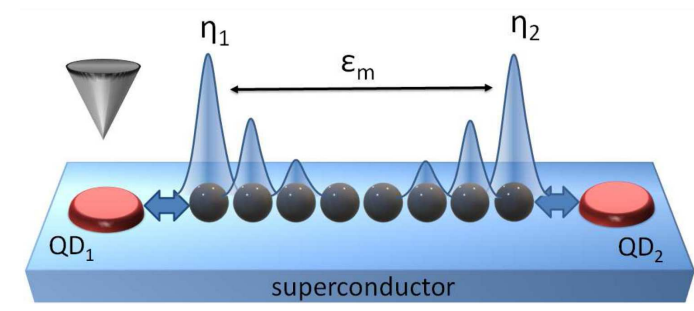

Fig. 1. The schematic illustration of the analyzed system. It consists of two quantum dots $\left(Q D_{1}\right.$ and $\left.Q D_{2}\right)$ coupled to opposite sides of a topological chain placed on top of conventional superconductor. Majorana quasiparticles are represented by self-hermitean operators $\eta_{1}$, $\eta_{2}$. The blue curves over the chain atoms illustrate schematically the probability amplitudes of the Majorana fermion wave functions. The overlap between edge states is represented by the term $\epsilon_{m}$. Grey cone over $Q D_{1}$ represents metallic STM tip.

hybrids can be achieved either by using depleting gates or by placing nonmagnetic adatoms at the end of a chain. For semiconducting nanowires, such hybrids can also be realized by selectively removing the $\mathrm{SC}$ layer from the end of the wire [8].

We inspect the features of long-range electron scattering mediated by edge states of the topological chain. Two bound states at the edges of the chain form one ordinary fermionic state. Therefore, the information on electrons injected into one end of a chain can be transferred into its opposite side, and further into a second quantum dot. As a consequence electron transmitted through one quantum dot can be scattered by an exchange of electrons between the second dot and nanowire. In other words, in our system we consider the topological chain as a bridge that couples two spatially separated quantum dots. Additionally, we assume that both QDs are coupled to external fermionic reservoirs but with different coupling strengths. In the schematic view (Fig. 1) STM tip suspended over one of the dots is considered as a strongly coupled fermionic reservoir. The influence of the environment on $Q D_{2}$ is taken into account as (very) weak interaction with some fermionic reservoir. The broadening of energy levels in nanoscopic heterostructures is controlled by tunneling rates to fermionic reservoirs. Therefore, in such circumstances disproportion in broadenings for each quantum dot obeys the conditions necessary for the appearance of Fano-like resonances. In absence of topological chain, the features of such quantum interference were predicted and observed in double quantum dot systems in both metallic [15-17] and superconducting environment [18-22].

\section{Model}

Considered heterostructure, including two quantum dots, is placed on top of $s$-wave superconductor. In our approach we will focus on energies deep inside the superconducting gap $(|\omega| \ll \Delta)$. In this, so-called deep superconducting atomic limit, the influence of a superconducting background on each quantum dot can be reduced to static sources and sinks of local pairs [2325]. Hamiltonian of each quantum dot $(j=1,2)$ placed in proximity of superconductor, in noncorrelated regime can be represented by

$$
\hat{H}_{Q D_{j}}=\sum_{\sigma} \epsilon_{j} \hat{d}_{j \sigma}^{\dagger} \hat{d}_{j \sigma}-\frac{1}{2} \Gamma_{S_{j}}\left(\hat{d}_{j \uparrow} \hat{d}_{j \downarrow}+\text { H.c. }\right),
$$

where $\hat{d}_{j \sigma}\left(\hat{d}_{j \sigma}^{\dagger}\right)$ stand for the creation (annihilation) operator of $\sigma$ spin electron on the $j$-th dot and $\Gamma_{S_{j}}$ represents the coupling constant between $j$-th dot and superconducting substrate. Quantum dots are connected to the opposite edges of the topological chain. Effectively each dot is tunnel-coupled to the corresponding Majorana bound state. Low energy physics of the chain and its coupling to the dots can be expressed by [26]

$$
\hat{H}_{M Q D}=\mathrm{i} \epsilon_{m} \hat{\eta}_{1} \hat{\eta}_{2}+\sum_{j=1,2} \lambda_{j}\left(\hat{d}_{j \uparrow} \hat{\eta}_{j}+\hat{\eta}_{j} \hat{d}_{j \uparrow}^{\dagger}\right),
$$

where self-hermitean operators $\hat{\eta}_{j}^{\dagger}=\hat{\eta}_{j}$ represent two edge states and $\epsilon_{m}$ stands for overlap between them. The last term of (2) stands for electron hopping between $j$-th dot and corresponding ( $j$-th) MBS. It is convenient to express Majorana states in terms of ordinary fermionic operators $\eta_{1}=\frac{1}{\sqrt{2}}\left(\hat{f}+\hat{f}^{\dagger}\right), \eta_{2}=\frac{\mathrm{i}}{\sqrt{2}}\left(\hat{f}-\hat{f}^{\dagger}\right)$. Using the local gauge transformation $\left(\hat{d}_{2} \rightarrow \mathrm{i} \hat{d}_{2}\right)$ and alternative dotchain coupling strengths $t_{j}=\frac{\lambda_{j}}{\sqrt{2}}$, Hamiltonian (2) can be rewritten in the following form

$$
\begin{aligned}
& \hat{H}_{M Q D}=\epsilon_{m} \hat{f}^{\dagger} \hat{f}+t_{1}\left(\hat{d}_{1 \uparrow}^{\dagger}-\hat{d}_{1 \uparrow}\right)\left(\hat{f}+\hat{f}^{\dagger}\right) \\
& \quad+t_{2}\left(\hat{d}_{2 \uparrow}^{\dagger}+\hat{d}_{2 \uparrow}\right)\left(\hat{f}-\hat{f}^{\dagger}\right) .
\end{aligned}
$$

Additionally, we assume that both dots are hybridized with ordinary fermionic reservoirs (e.g. with metallic STM tip). Such reservoirs are represented by

$$
\hat{H}_{N_{j}}=\sum_{k, \sigma} \xi_{j k} C_{j k \sigma}^{\dagger} C_{j k \sigma},
$$

where energy of the electrons $\xi_{j k}$ are measured with respect to their chemical potential $\mu_{j}$. Electron tunneling between $j$-th dot and $j$-th reservoir is represented by

$$
\hat{H}_{T_{j}}=\sum_{k, \sigma} V_{j k}\left(C_{j k \sigma} \hat{d}_{j \sigma}^{\dagger}+H . c .\right) \text {. }
$$

In further calculations we will use the wide band limit coupling constant $\Gamma_{N_{j}}=2 \pi \sum_{k}\left|V_{j k}\right| \delta\left(\omega-\xi_{k}\right)$ between $j$-th dot and corresponding reservoir.

The whole heterostructure (depicted in Fig. 1) is given by following Hamiltonian

$$
\hat{H}_{\text {sys }}=\sum_{j}\left(\hat{H}_{Q D_{j}}+\hat{H}_{N_{j}}+\hat{H}_{T_{j}}\right)+\hat{H}_{M Q D} .
$$

Information on density of states for each quantum dot is encoded in Fourier transform of single-particle retarded Green's function $G_{11}^{j}\left(\omega+\mathrm{i} 0^{+}\right)=\left\langle\left\langle\hat{d}_{j \uparrow} \hat{d}_{j \uparrow}^{\dagger}\right\rangle\right\rangle$. The abovepresented model is exactly solvable (as long as we focus on the noncorrelated regime) and solution can be provided e.g. by the Green's function equation of motion technique (EOM). 
Introducing auxiliary factors

$$
a_{j}(\omega)=\omega-\epsilon_{j}+\mathrm{i} \Gamma_{N_{j}}-\frac{\Gamma_{S_{j}}}{\omega+\epsilon_{j}+\mathrm{i} \Gamma_{N_{j}}} \quad \text { and } \quad b_{j}(\omega)=-\left[a_{j}(-\omega)\right]^{*},
$$

this method yields

$$
G_{11}^{j}(\omega)=\frac{\left(2 t_{j}^{2}-b_{j} \omega\right)\left(a_{\bar{j}} b_{\bar{j}} \omega-2 t_{\bar{j}}^{2}\left(a_{\bar{j}}+b_{\bar{j}}\right)\right)+a_{\bar{j}} b_{j} b_{\bar{j}} \epsilon_{m}^{2}}{\left(-a_{j} b_{j} \omega+2 a_{j} t_{j}^{2}+2 b_{j} t_{j}^{2}\right)\left(a_{\bar{j}} b_{\bar{j}} \omega-2 t_{\bar{j}}^{2}\left(a_{\bar{j}}+b_{\bar{j}}\right)\right)+a_{j} a_{\bar{j}} b_{j} b_{\bar{j}} \epsilon_{m}^{2}},
$$

where $\bar{j}=1$ if $j=2$ (and counterwise $\bar{j}=2$ if $j=1$ ). Density of states for each dot is represented by imaginary part of corresponding Green's function

$$
\rho_{j}(\omega)=-\frac{1}{\pi} \operatorname{Im}\left(G_{11}^{j}\left(\omega+\mathrm{i} 0^{+}\right)\right) .
$$

\section{Signatures of scattering}

To properly describe our results let us first recall some findings on proximity effect and its interplay with Majorana modes. When QD is connected to $s$-wave superconductor it adopts some SC properties. As a result, a single-particle level $(\epsilon)$ evolves into two quasiparticle Andreev states (also called Yu Shiba Rusinov states) $[23,24]$. In the deep superconducting atomic limit, these states are located at $\pm \sqrt{\left(\frac{1}{2} \Gamma_{S}\right)^{2}+\epsilon^{2}}$, where $\Gamma_{S}$ denote coupling strength between quantum dot and superconducting electrode. If such QD is connected to one end of a topological chain (with coupling strength $t$ ), two trivial Andreev states are accompanied by two "molecular" levels appearing as a result of the coalescence of Andreev quasiparticle states with Majorana edge states [27-29]. This additional states are located at $\pm \sqrt{\left(\frac{1}{2} \Gamma_{S}\right)^{2}+\epsilon^{2}+(2 t)^{2}}$. The broadening of all these states is controlled by coupling of the dot to fermionic reservoirs (such as metallic STM tip).

In a considered system, with two QD's coupled to opposite edges of a topological wire, the metallic STM tip is suspended over one of the dots. Tip-dot coupling strength is given by wide-band limit coupling constant $\Gamma_{N_{1}}=\sum_{k} V_{k N 1} \delta\left(\omega-\xi_{k N 1}\right)$ which we use as the energy unit. We assume that the second dot weakly interacts with some external fermionic reservoir $\left(\Gamma_{N 2}=0.001 \Gamma_{N 1}\right)$. As a result, all four states appearing in $Q D_{1}$ (two trivial ABS and two molecular levels) are significantly broadened while states at $Q D_{2}$ remain quasi-discrete.

In Figs. 2 and 3 we present the local density of states (LDOS) of $Q D_{1}$ and $Q D_{2}$. The initial level of both dots were established as zero $\left(\epsilon_{1}=\epsilon_{2}=0\right)$. We assumed that coupling between dots and the superconducting substrate is slightly higher than the coupling of $Q D_{1}$ to STM tip $\left(\Gamma_{S_{1}}=\Gamma_{S_{2}}=2 \Gamma_{N_{1}}\right)$. This reflects the assumption that both dots are placed directly

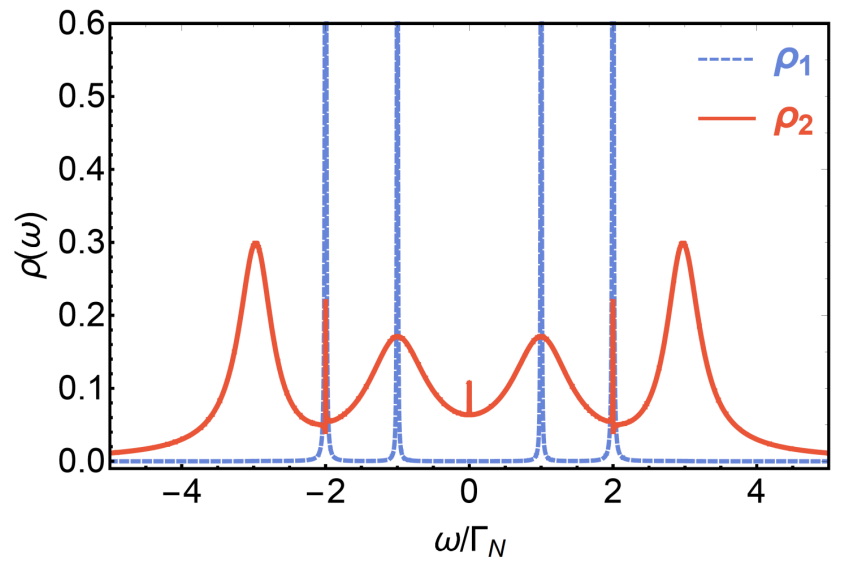

Fig. 2. Density of states for $Q D_{1}$ (solid red line) and $Q D_{2}$ (dashed blue line) obtained for following model parameters $\Gamma_{S_{1}}=\Gamma_{S_{2}}=2 \Gamma_{N_{1}}, \Gamma_{N_{2}}=0.001 \Gamma_{N_{1}}, t_{1}=$ $\sqrt{2} \Gamma_{N_{1}}, t_{2}=\sqrt{3} / 2 \Gamma_{N_{1}}, \epsilon_{m}=0.1 \Gamma_{N_{1}}$.

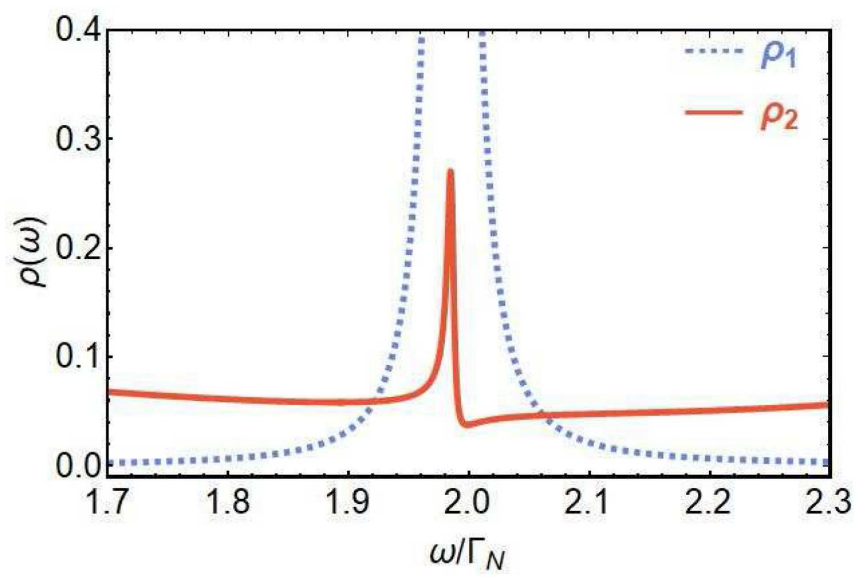

Fig. 3. Closeup of a resonant feature presented in Fig. 2 obtained for the same model parameters.

on top of the superconducting substrate. Coupling of each dot to corresponding edges of a chain was set to $t_{1}=\sqrt{3} / 2, t_{2}=\sqrt{2}$. Additionally, we assume small but finite overlap between the edge states $\epsilon_{m}=0.1 \Gamma_{N_{1}}$. Although true Majorana zero mode is realized with vanishing $\epsilon_{m}$, in physical systems finite length of a chain enforces a finite overlap between these states. 


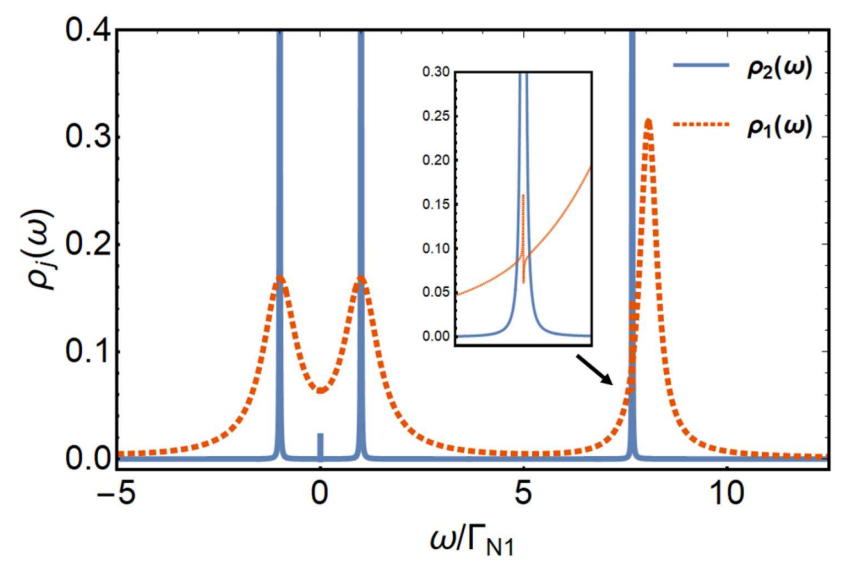

Fig. 4. Density of states for $Q D_{1}$ (dashed red line) and $Q D_{2}$ (solid blue line) obtained for following model parameters $\Gamma_{S_{1}}=\Gamma_{S_{2}}=2 \Gamma_{N_{1}}, \Gamma_{N_{2}}=0.001 \Gamma_{N_{1}}$, $t_{1}=4 \Gamma_{N_{1}}, t_{2}=3.8 \Gamma_{N_{1}}, \epsilon_{m}=0.02 \Gamma_{N_{1}}$. Inset shows the closeup of a resonant interferometric feature near molecular level of $Q D_{2}$.

Using such assumptions we obtain two ordinary and two molecular states in the density of states of each dot. According to the description above, the states on $Q D_{1}$ are wide while the states on $Q D_{2}$ remain narrow (quasidiscrete). Trivial ABS states for both dots appear at $\omega= \pm \Gamma_{N_{1}}$. Molecular states for $Q D_{1}$ appear at $\omega= \pm 3 \Gamma_{N_{1}}$, while for $Q D_{2} \omega= \pm 2 \Gamma_{N_{1}}$. Additionally in LDOS of a $Q D_{1}$ we predict well-pronounced asymmetric features characteristic for scattering on a narrow level. Such result indicates that the electrons transferred through one dot "feel" the existence of discrete levels on the opposite side of a topological chain. Such quantum interference between broad levels of $Q D_{1}$ and discrete levels of $Q D_{2}$ produces the characteristic asymmetric Fano-like profiles in LDOS of $Q D_{1}$. Remarkably, we observe such features only for energies equal to energy of molecular levels of $Q D_{2}$ (i.e. $\omega= \pm \sqrt{\left(\frac{1}{2} \Gamma_{S_{2}}\right)^{2}+\left(2 t_{2}\right)^{2}}$ ) and not for trivial Andreev states. This indicates that only molecular states of $Q D_{2}$ take part in the scattering, while information on trivial Andreev states is not transferred through topological chain. One should note that the position of molecular levels of $Q D_{2}$, and thus position of interference patterns observed in $Q D_{1}$ can be tuned either by changing dot-chain coupling strength $\left(t_{2}\right)$ or more realistically by application of additional gate voltage that manipulates initial dot level $\left(\epsilon_{2}\right)$. We find that characteristic features provided by long-range scattering appear only for a finite overlap of edge states $\left(\epsilon_{m}\right)$. Although the finite overlap between edge states is perfectly reasonable from the experimental point of view (as chain's length must also be finite), such restriction does not obey the conditions for a true Majorana zero mode. Nevertheless, with careful tuning of model parameters, we predict that asymmetric features should be noticeable even when the overlap between edge states is over two magnitude orders lower than couplings between dots and chain (see Fig. 4).

\section{Conclusions}

In the presented setup we predicted the appearance of interferometric Fano-like profiles, provided by information transfer through edge states of the topological chain. We obtained that the position of such features, appearing on one side of a chain (one dot) can be tuned by manipulation of parameters associated locally to the opposite side of the nanowire. Predicted resonances appear for small but finite overlap of nanochain edge states.

\section{Acknowledgments}

The research has been conducted in the framework of the project implemented in 2018-2021, entitled "Analysis of nanoscopic systems coupled with superconductors in the context of quantum information processing". No GB/5/2018/209/2018/DA funded by the Ministry of National Defence Republic of Poland.

\section{References}

[1] A.Y. Kitaev, Phys. Usp. 44, 131 (2001).

[2] L. Fu, Phys. Rev. Lett. 104, 056402 (2010).

[3] V. Mourik, K. Zuo, S.M. Frolov, S.R. Plissard, E.P.A.M. Bakkers, L.P. Kouwenhoven, Science. 336, 1003 (2012).

[4] O. G'ul, H. Zhang, J.D.S. Bommer, M.W.A. de Moor, D. Car, S.R. Plissard, E.P.A.M. Bakkers, A. Geresdi, K. Watanabe, T. Taniguchi, L.P. Kouwenhoven, Nat. Nanotechnol. 13, 192 (2018).

[5] C.X. Liu, J.D. Sau, T.D. Stanescu, S. Das Sarma, Phys. Rev. B 96, 075161 (2017).

[6] R. Pawlak, M. Kisiel, J. Klinovaja, T. Meier, S. Kawai, T. Glatzel, D. Loss, E. Meyer, npj Quantum Info 2, 16035 (2016).

[7] H. Kim, A. Palacio-Morales, T. Posske, L. Rózsa, K. Palotas, L. Szunyogh, M. Thorwart, R. Wiesendanger, Sci. Adv. 4, 5251 (2018).

[8] M.T. Deng, S. Vaitiekenas, E.B. Hansen, J. Danon, M. Leijnse, K. Flensberg, J. Nygard, P. Krogstrup, C.M. Marcus, Science 354, 1557 (2016).

[9] B.E. Feldman, M.T. Randeria, J. Li, S. Jeon, Y. Xie, Z. Wang, I.K. Drozdov, B.A. Bernevig, A. Yazdani, Nature Phys. 13, 286 (2017) .

[10] J. Nilsson, A.R. Akhmerov, C.W.J. Beenakker, Phys. Rev. Lett. 101, 120403 (2008).

[11] P. Wang, Y. Cao, M. Gong, G. Xiong, X.-Q. Li, Europhys. Lett. 103, 57016 (2013).

[12] P. Wang, Y. Cao, M. Gong, S.-S. Li, X.-Q. Li, Phys. Lett. A 378, 937 (2014).

[13] J. Liu, F.-C. Zhang, K.T. Law, Phys. Rev. B 88, 064509 (2013).

[14] C.J. Bolech. E. Demler, Phys. Rev. Lett. 98, 237002 (2007).

[15] M.E. Torio, K. Hallberg, A.H. Ceccatto, C.R. Proetto, Phys. Rev. B 65, 085302 (2002). 
[16] B.R. Bulka, P. Stefanski,Phys. Rev. Lett. 86, 5128 (2001).

[17] K.P. Wojcik, I. Weymann, Phys. Rev. B 90, 115308 (2014).

[18] A.M. Calle, M. Pacheco, G.B. Martins, V.M. Apel, G.A. Lara, P.A. Orellana, J. Phys.: Condens. Matter 29, 135301 (2017).

[19] J. Baranski, T. Domanski, Phys. Rev. B 84, 195424 (2011).

[20] J. Baranski, T. Domanski, Phys. Rev. B 85, 205451 (2012).

[21] J. Baranski, T. Domanski, Chin. Phys. B 24, 017304 (2015).

[22] S. Glodzik, K.P. Wojcik, I. Weymann, T. Domanski, Phys. Rev. B 95, 125419 (2017).

[23] J. Baranski, T. Domanski, J. Phys.: Condens. Matter 25, 435305 (2013).
[24] J. Bauer, A. Oguri, A.C. Hewson, J. Phys.: Condens. Matter 19, 486211 (2008).

[25] T. Domanski, J. Baranski, M. Zapalska, Phil. Mag. 95, 538 (2015).

[26] D. E. Liu, M. Cheng, R. Lutchyn, Phys. Rev. B 91 , 081405 (2015).

[27] S. Hofman, D. Chevallier, D. Loss, J. Klinovaja, Phys. Rev. B 96, 045440 (2017).

[28] A. Ptok, A. Kobialka, T. Domanski, Phys. Rev. B 96, 195430 (2017)

[29] G. Gorski, J. Baranski, I. Weymann, T. Domanski, Sci. Rep. 8, 15717 (2018).

[30] J. Baranski, A. Kobialka, T. Domanski, J. Phys.: Condens. Matter 29, 075603 (2017). 